\title{
GRAINE project: Cosmic Gamma-ray Observation by Balloon-Borne Telescope with Nuclear Emulsion
}

\author{
Shigeki Aoki ${ }^{1}$ \\ Kobe University \\ Kobe 657-8501 Japan \\ E-mail: aoki@kobe-u.ac.jp
}

for GRAINE collaboration

Kobe University, Kobe 657-8501, Japan

Nagoya University, Nagoya 464-8602, Japan

ISAS/JAXA, Sagamihara 252-5210, Japan

Aichi University of Education, Kariya 448-8542, Japan

Okayama University of Science, Okayama 700-0005, Japan

GRAINE, which stands for Gamma-Ray Astro-Imager with Nuclear Emulsion is a new gammaray observation project with balloon-borne emulsion telescope. Nuclear emulsion can determines incident gamma-ray angle measuring electron positron track right after the conversion with small material thickness (.002 radiation length par film). Thanks of this small thickness, angular resolution can reach $0.1^{\circ}$ for $1 \mathrm{GeV}$ gamma-ray $\left(1.0^{\circ}\right.$ for $\left.100 \mathrm{MeV}\right)$ and it can be sensitive to polarization. GRAINE could bring precise observation of gamma-ray sources especially on the galactic plane by repeating long-duration flights with large aperture $\left(10 \mathrm{~m}^{2}\right)$ emulsion telescope.

In May 2015, we launched an emulsion telescope of $0.38 \mathrm{~m}^{2}$ aperture area from Alice Springs, Australia in order to demonstrate the overall imaging performance. Flight duration time was about 14.4 hours with 11.5 hours level flight at $37 \mathrm{~km}$ altitude including 6 hours with the Vela pulsar in the field of view. Latest results and future plans are described.

The 3rd International Symposium on "Quest for the Origin of Particles and the Universe"

5-7 January 2017

Nagoya University, Japan

\section{${ }^{1}$ Speaker}




\section{Introduction}

Cosmic gamma-ray/X-ray bring us direct information of high energy phenomena in the universe. Since 2008, the Large Area Telescope on the Fermi satellite (Fermi-LAT) has made huge progress in the sub-GeV/GeV energy region of gamma-ray astronomy. However, observation around the galactic plane has difficulty because angular resolution is not good enough to resolve crowded region with higher back ground flux originated from the galactic diffused gamma-rays. GRAINE (Gamma-Ray Astro-Imager with Nuclear Emulsion) is a new gamma-ray observation project with balloon-borne emulsion telescope. GRAINE could bring precise observation of gamma-ray sources especially on the galactic plane by repeating long-duration flights with large aperture $\left(10 \mathrm{~m}^{2}\right)$ emulsion telescope.

\section{Emulsion Gamma-Ray Telescope}

Nuclear emulsion is a powerful tracking device that can record threedimensional tracks of charged particles with precise position resolution $(<1 \mu \mathrm{m})$. Nuclear emulsion can determines incident gamma-ray angle measuring electron positron track right after the conversion with small material thickness $(.002$ radiation length par film). Thanks of this small thickness, angular resolution can reach $0.1^{\circ}$ for $1 \mathrm{GeV}$ gamma-ray $\left(1.0^{\circ}\right.$ for $100 \mathrm{MeV}$ ) as shown in Fig.1. Measuring azimuthal distribution of the opening angle of the pair of electron and positron, polarization of the incident gamma-ray can be determined. Fig. 2 shows the azimuthal angle distribution of the selected electronpositron pairs converted by gamma-ray beam at LEPS (Laser Electron Photon beam line at Spring-8) in Japan. Best fit amplitude and polarization direction were 0.14 and $110^{\circ}$ respectively, and they are consistent with expectation.[1]

A schematic view of the emulsion gamma-ray telescope is shown in Fig.3.

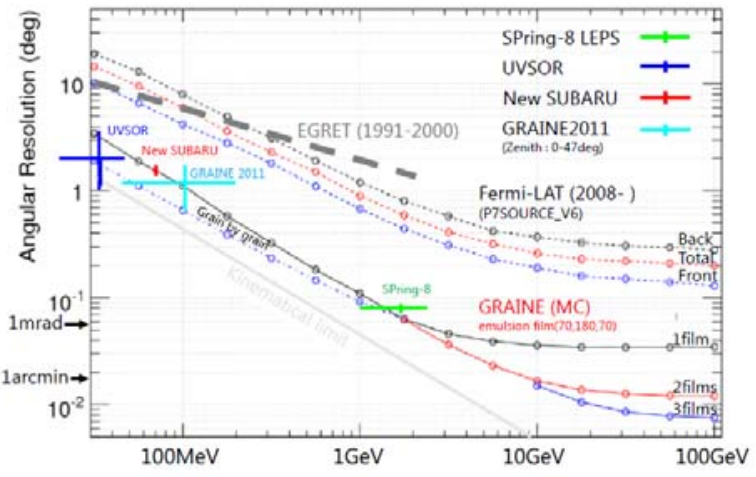

Fig.1. Angular resolution of emulsion gamma-ray telescope (solid lines show simulation results and dots with error bars show experimental results), Fermi-LAT (dotted lines), and EGRET (a broken line).

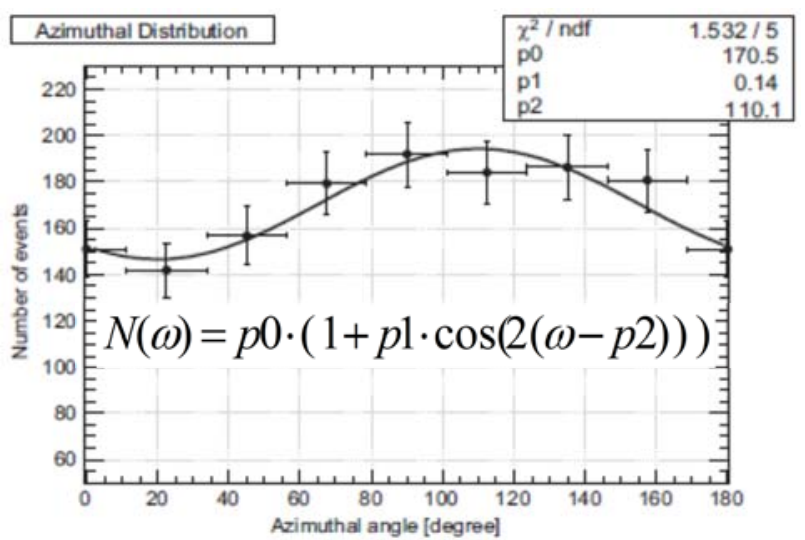

Fig.2. Azimuthal angle distribution of the selected electron-positron pairs. (vertical srror bars show statistical error only)[1] The telescope consists of a converter, time stamper, calorimeter and an attitude monitor. The converter consists of a stack of emulsion films. We use emulsion film that has emulsion layers coated on both sides of plastic base. The start of electron pair creation is detected at the converter. The time stamper consists of multi-stage shifter which gives timing information to converter events, normally lacking in emulsion detection. The calorimeter consists of a stack of emulsion films with metal plates. Gamma-ray energies below 
several $\mathrm{GeV}$ are measured in the converter by measuring multiple coulomb scattering. The attitude monitor consists of a star camera. By combining the attitude monitor information and the event timing, the gamma-ray direction relative to the celestial sphere is determined.

The attitude of the balloon-borne telescope changes at a rate of approximately a milli-radian per second. Thus milli-radian angular resolution requires a time resolution less than a second. We developed a multi-stage shifter as the time stamper.[2] The multi-stage shifter consists of cyclically sliding individual emulsion films relative to each other. A multi-stage shifter is similar to an analog clock which shows 12 hours with second accuracy by several hands moving with individual cycles. By increasing number of stages with a shorter cycle, the time resolution is improved. Multi-stage shifter achieves time-stamping by using a simple design, which is compact, light, high voltage free, low power and no dead-time.

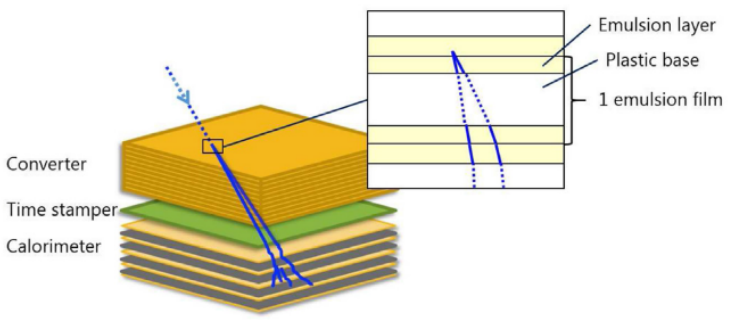

Fig. 3. Schematic view of emulsion gamma-ray telescope

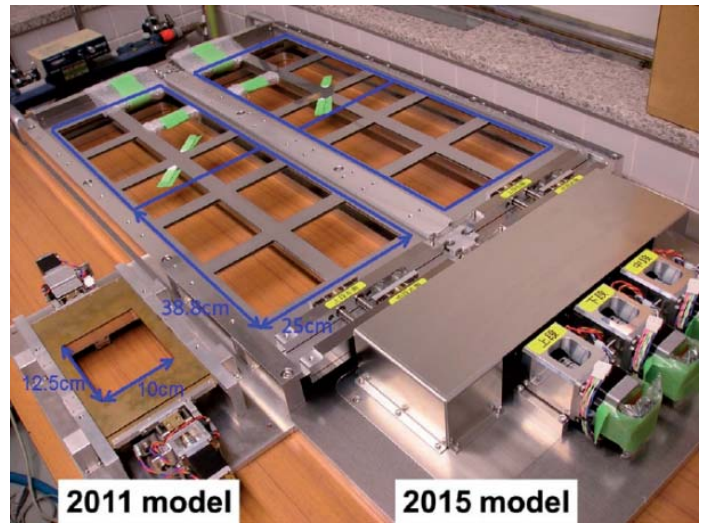

Fig. 4. Flight models of the multi-stage shifter. The left model $\left(125 \mathrm{~cm}^{2}\right.$ aperture area) was used in the 2011 balloon experiment. The right model $\left(3800 \mathrm{~cm}^{2}\right.$ aperture area) was used in the 2015 balloon experiment.

\section{Balloon Experiment}

In June 2011, we performed the first balloon borne emulsion telescope experiment at JAXA Taiki Aerospace Research Field in Hokkaido, Japan in order to confirm our strategy. $12.5 \times 10 \mathrm{~cm}^{2}$ aperture area telescope was flown 4.3 hours including 1.6 hours level flight at $34.7 \mathrm{~km}$ altitude.[3] Systematic detection, energy reconstruction, and timestamping of gamma-ray events were performed across the whole area of the emulsion film, up to $45^{\circ}$ incident zenith angle, down to $50 \mathrm{MeV}$ gamma-ray energy, with $97 \%$ detection reliability. We confirmed all the components of the telescope worked properly under balloon experiment environment (low temperature, low air pressure). From flight data, the atmospheric gamma-ray flux was measured in the energy range 50$300 \mathrm{MeV}$ and obtained a first understanding of the cosmic gamma-ray background. By combining the attitude data, we demonstrated a procedure for determining the gamma-ray arrival direction in celestial coordinates.

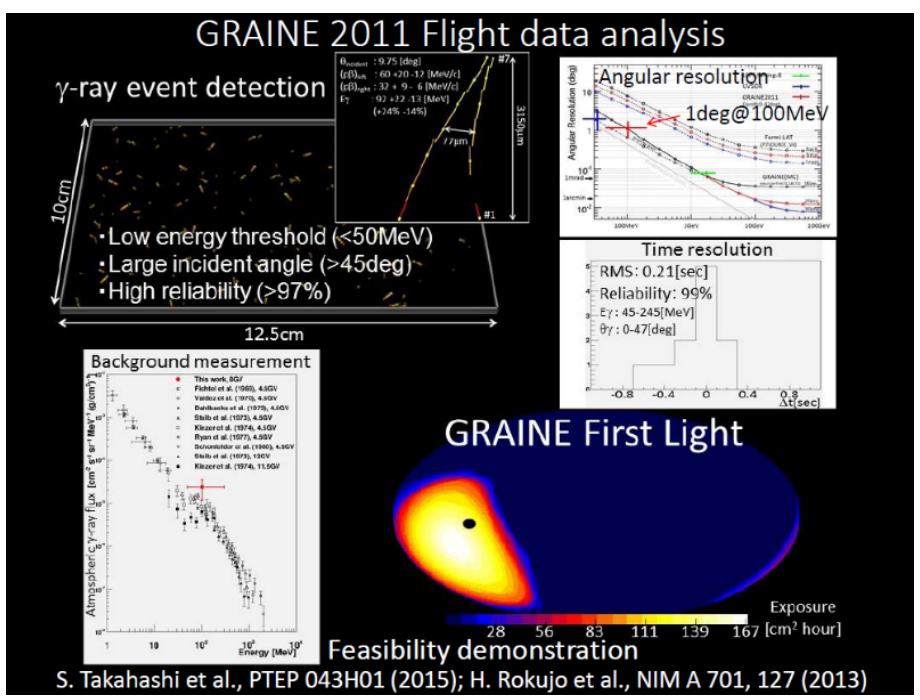

Fig. 5. Outcomes from GRAINE 2011 balloon experiment 
In May 2015, we performed 2nd balloon-borne experiment in Alice Springs, Australia, in order to demonstrate overall imaging performance of our telescope.[4,5] The emulsion telescope that has the aperture area of $0.38 \mathrm{~m}^{2}$ was employed in this experiment as shown in Fig.6. Flight duration time was about 14.4 hours with 11.5 hours level flight at $37 \mathrm{~km}$ altitude including 6 hours with the Vela pulsar in the field of view.
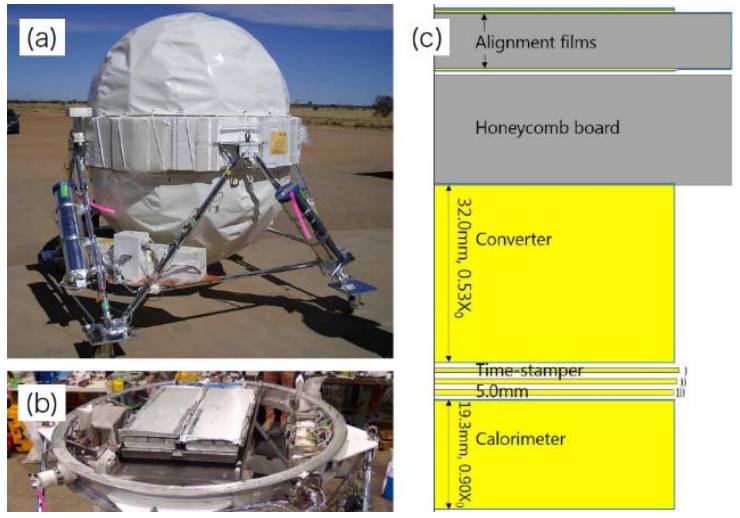

Fig. 6. (a) Picture of gondola. A spherical container (the inside diameter is $1.6 \mathrm{~m}$ ) is a balloon-style pressure vessel. Three star cameras as the attitude monitors were mounted on the trusses (one of them was in the other side).

(b) Inside view of the pressure vessel. At the center of a ring, the emulsion telescope was put. The total aperture area of four units was 3800 $\mathrm{cm} 2$.

(c) Cross sectional view of the emulsion telescope. It consists of alignment films, the converter, and time stamper, and the calorimeter.

Fig. 6 shows the picture of the pressure vessel and gondola with emulsion telescope mounted at center. Cross sectional view of the telescope is also shown. Detailed description of the flight data analysis for gamma-ray event is shown elsewhere.[6,7]

After the analysis of tracks reconstructed at time stamper each emulsion track has time information of penetrating. Therefore track rate of every second during flight can be extracted as shown in Fig.7. With ascension of altitude, Pfotzer maximum is confirmed and the rate becomes stable during level flight. In the track rate many spikes are detected. Among these high rate events, we found many events with tracks conversing into one point. Most of them seem hadronic interaction in the converter caused by high energy cosmic ray particle. Since these tracks are known to have same timing. We could determine time resolution based on flight data.

Many of these hadronic interactions are often associate gamma-ray from neutral pion. Since these gamma-ray become background for cosmic gamma-ray observation, we should eliminate these gamma-ray event detecting parent hadronic interaction vertex aligned on the direction of gamma-ray. On the other hand, these gamma-ray event become good calibration source to determine angular resolution of gamma-ray direction. Fig. 8 shows the result of the measurement.

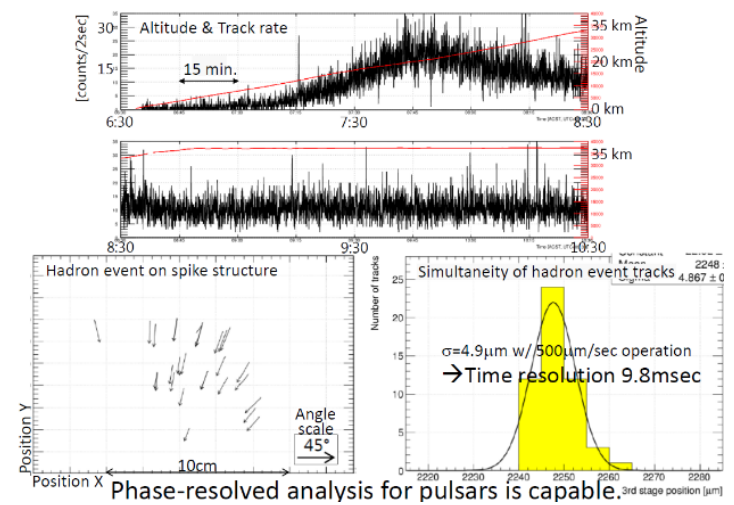

Fig. 7. (top)Track rate by time stamper analysis. (left)Hadronic interaction tagged by high track rate. (right)Time distribution of daughter tracks of hadronic interaction shows $9.8 \mathrm{msec}$ time resolution

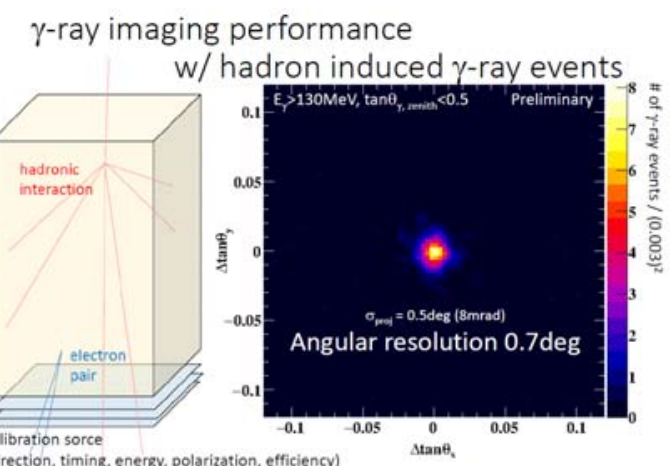

High $\gamma$-ray imaging performance was being obtained.

Fig. 8. Angular resolution determined by gamma-ray from hadronic interaction 


\section{Outlook for Large Scale Scientific Observation Flight}

Emulsion film has good advantage because enlargement of film itself is relatively simple. For scientific observation flight, we have to enlarge aperture area keeping the balloon capable weight limit. Because the weight of converter is directly coupled to sensitivity, we have to reduce the weight of other part. New design of weight saved multi stage shifter and large scale pressure vessel are under study as shown in Fig.9 and Fig.10.

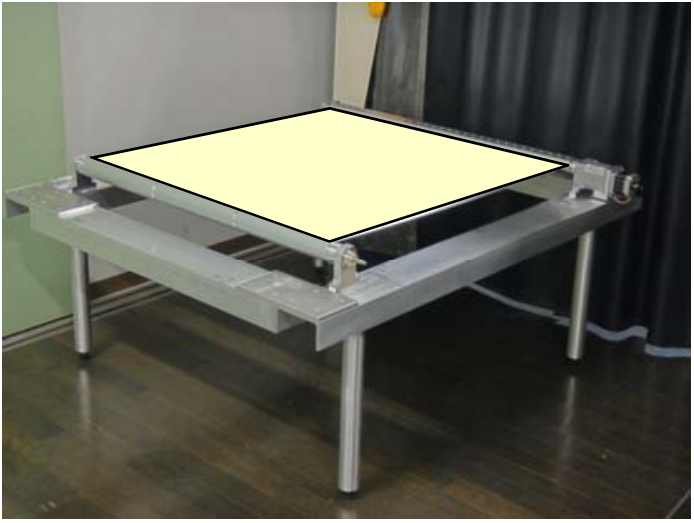

Fig. 9. Roller type shifter for weigh saving

In April 2018, we will have next opportunity of balloon experiment at Alice Springs, Australia in order to demonstrate imaging of Astronomical object (Vela pulser etc.). Enlarging the aperture area and repeating the large scale scientific observation with long-duration balloon flights, we expect various out come as shown in Fig.11.

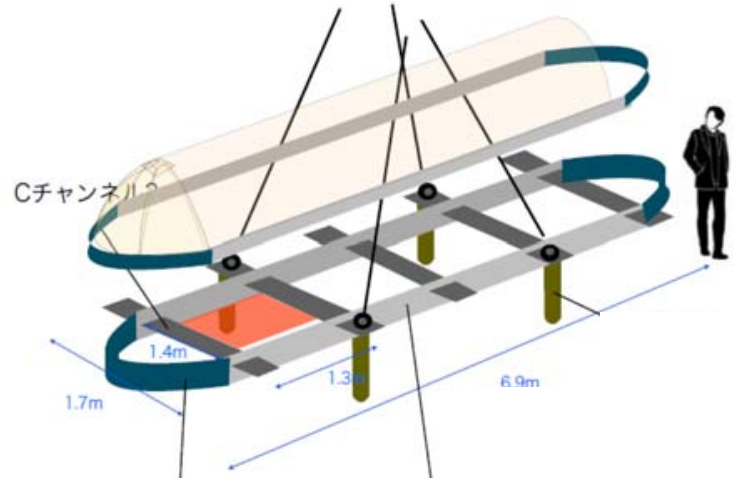

Fig. 10. $5 \mathrm{~m}^{2}$ gondola and pressure vessel

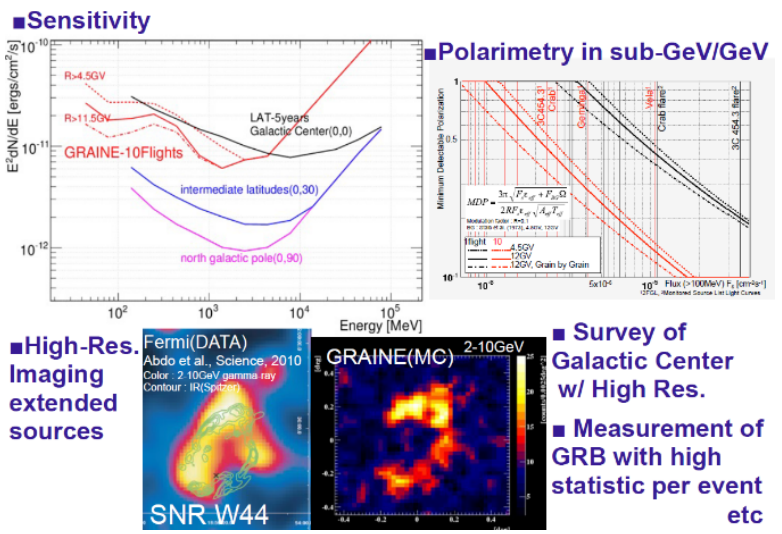

Fig. 11. Expected outcome after large scale scientific observation flight

\section{References}

[1] K. Ozaki et al., Demonstration of polarization sensitivity of emulsion-based pair conversion telescope for cosmic gamma-ray polarimetry, NIM A833 (2016) 165-168

[2] S. Takahashi et al., Time stamp technique using a nuclear emulsion multi-stage shifter for gammaray telescope, NIM A620 (2010) 192-195

[3] S. Takahashi et al., GRAINE project: The first balloon-borne, emulsion gamma-ray telescope experiment, PTEP 2015 (2015) 043H01

[4] K. Ozaki et al., Development of new-type nuclear emulsion for a balloon-borne emulsion gammaray telescope experiment, JINST 10 (2015) no.12, P12018

[5] S. Takahashi et al., GRAINE 2015, a balloon-borne emulsion $\gamma$-ray telescope experiment in Australia, PTEP 2016 (2016) 073F01

[6] H. Rokujo and GRAINE collaboration, GRAINE balloon experiment in 2015:Precise observation of cosmic gamma rays by high-resolution emulsion telescope, ISVHECRI 2016

[7] H. Rokujo, GRAINE Project: balloon-borne emulsion gamma-ray telescope, KMI2017 